\title{
Short-Range Atomic Ordering in Amorphous Ion-Tracks in Pyrochlores
}

\author{
R. Sachan ${ }^{1}$, B. Liu ${ }^{2}$, D. Aidhy ${ }^{1}$, Y. Zhang ${ }^{1,2}$, M. F. Chisholm ${ }^{1}$ and W. J. Weber ${ }^{2,1}$ \\ ${ }^{1}$ Materials Science and Technology Division, Oak Ridge National Laboratory, Oak Ridge, \\ Tennessee 37831 \\ ${ }^{2}$ Department of Materials Science and Engineering, University of Tennessee, Knoxville, TN \\ 37996
}

Pyrochlore structured complex oxides $\left(\mathrm{A}_{2} \mathrm{~B}_{2} \mathrm{O}_{7}\right)$ have attracted attention due to its unique physical, chemical and electrical properties. Due to its radiation tolerance, pyrochlores are seen as a candidate for application such as inert matrix fuels, nuclear waste immobilization and geological age-dating.[1,2] However, radiation under such extreme conditions introduces phase transformation in the ordered pyrochlore structure, which is critical to understand for such applications. High energy ion irradiation ( $\mathrm{MeV}$ to $\mathrm{GeV}$ ) on pyrochlores is seen as an important experiment to understand the phase transformations which take place due to localized (nanoscale) energy transfer to the lattice in extremely short time period ( ns).[3]

In the present work, $\mathrm{Gd}_{2} \mathrm{Ti}_{2} \mathrm{O}_{7}$ is irradiated with high energy ions to form nanoscale amorphous tracks along the ion penetration path. It has been previously reported that a concentric shell of disordered crystalline phase of defect fluorite is formed around the amorphous ion track in $\mathrm{Gd}_{2} \mathrm{Zr}_{\mathrm{x}} \mathrm{Ti}_{(1-\mathrm{x})} \mathrm{O}_{7}$ pyrochlores.[4] In the case of $\mathrm{Gd}_{2} \mathrm{Ti}_{2} \mathrm{O}_{7}$, there are studies reporting similar formation of a concentric defect-fluorite phase. However, atomic scale characterization and its effect on electronic structure of material has not yet been understood which is important for performance of such materials. Here, we report the detailed study of concentric defect fluorite phase existing around amorphous ion track by using high-angle annular dark field (HAADF) imaging and electron energy-loss spectroscopy (EELS) in an aberration corrected scanning transmission electron microscope (STEM).

Here, a (HAADF) image of an ion track in $\mathrm{Gd}_{2} \mathrm{Ti}_{2} \mathrm{O}_{7}$ pyrochlore lattice oriented in [110] zone axis is shown in Fig. 1(a). The ion track was created by $2.2 \mathrm{GeV}$ Au ion irradiation. The image analysis based on the atomic column contrast reveals that a distinct crystalline phase with thickness of 2-3 atomic planes $(0.5-1 \mathrm{~nm})$ forms at the periphery of the ion track. This phase is identified as defect fluorite phase which is a disordered crystalline derivative of the ordered pyrochlore phase. The contrast variation seen in amorphous region is due to tilting of ion track from perfect normal view. To understand the electronic structure, electron energy-loss spectroscopy (EELS) is performed. Fig. 1(b) and (c) show the Ti L-edge (456 eV) and O K-edge $(532 \mathrm{eV})$ respectively from ordered crystalline and amorphous region. In an important observation, the Ti L-edge in the amorphous ion track features crystal field splitting similar to the one from crystalline region. This result indicates the presence of short-range ordering in the Ti-O octahedra in amorphous phase of $\mathrm{Gd}_{2} \mathrm{Ti}_{2} \mathrm{O}_{7}$. In addition to that, the shift in the $\mathrm{e}_{\mathrm{g}}$ and $\mathrm{t}_{2 \mathrm{~g}}$ peaks in both $\mathrm{L}_{3}$ and $\mathrm{L}_{2}$ shows the evidence of Ti-O octahedral distortion in the amorphous phase. Fig. 1(c) shows that the pre-peak merges with the main peak and results in a significant 
broadening of the $\mathrm{O}$ K-edge. These results are consistent with density functional theory (DFT) calculations that show overlapping of the density of states for the amorphous state.

\section{References:}

1. K. E. Sickafus et al., Science 289 (2000) 748.

2. J. Zhang et al., J. Mater. Res., 25 (2010) 1344.

3. J. Wang, J. Phys.: Condens. Matter, 25 (2013) 135001.

4. M. Lang et al., Nat. mater. 8 (2009) 793.

5. This work was sponsored by US Department of Energy, Office of Science, Basic Energy Sciences, Materials Sciences and Engineering Division.

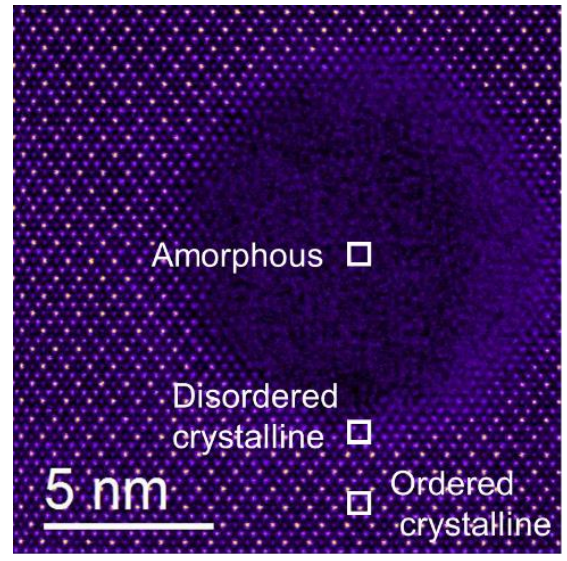

(a)

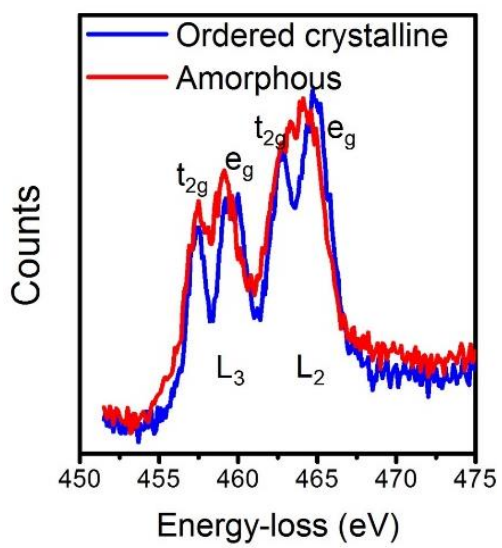

(b)

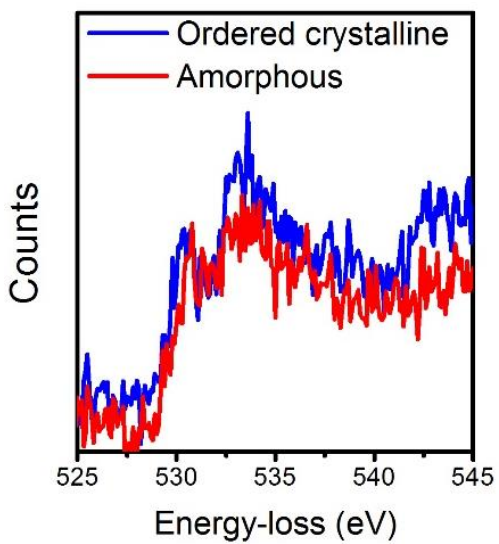

(c)

Fig. 1 (a) A high angle annular dark field (HAADF) image of an ion-track in $\mathrm{Gd}_{2} \mathrm{Ti}_{2} \mathrm{O}_{7}$ matrix showing amorphous, disordered crystalline and ordered crystalline region. (b) Ti L-edge from amorphous and ordered region, featuring presence of crystal field splitting, thus short-range ordering in amorphous phase. (c) O K-edge from amorphous and crystalline region. 